ISSN 1936-5098

CAE Working Paper \#08-10

\title{
Markov Processes Generated by Random Iterates of Monotone Maps: Theory and Applications
}

by

Mukul Majumdar

December 2008 


\title{
Markov Processes Generated by Random Iterates of Monotone Maps: Theory and Applications
}

\author{
Mukul Majumdar \\ Department of Economics \\ Uris Hall, Fourth Floor \\ Cornell University \\ Ithaca, New York 14853-7601 \\ e-mail address: mkm5@cornell.edu
}

June 18, 2008

\begin{abstract}
The paper is a review of results on the asymptotic behavior of Markov processes generated by i.i.d. iterates of monotone maps. Of particular importance is the notion of splitting introduced by Dubins and Freedman (1966). Some extensions to more general frameworks are outlined, and, finally, a number of applications are indicated.

*This paper is primarily based on my research with Professor Rabi Bhattacharya over a number of years. No formal acknowledgement to his influence is adequate. Thanks are due to the referee for a careful reading and suggestions on expository changes.
\end{abstract}




\section{Introduction}

This paper is an impressionistic overview of some results on Markov processes that arise in the study of a particular class of random dynamical systems. A random dynamical system is described by a triplet $(S, \Gamma, Q)$ where $S$ is the state space (for example, a metric space), $\Gamma$ an appropriate family of maps on $S$ into itself (interpreted as the set of all possible laws of motion) and $Q$ is a probability measure on (some $\sigma$-field of) $\Gamma$.

The evolution of the system can be described as follows: initially, the system is in some state $x$; an element $\alpha_{1}$ of $\Gamma$ is chosen randomly according to the probability measure $Q$ and the system moves to a state $X_{1}=\alpha_{1}(x)$ in period one. Again, independently of $\alpha_{1}$, an element $\alpha_{2}$ of $\Gamma$ is chosen according to the probability measure $Q$ and the state of the system in period two is obtained as $X_{2}=\alpha_{2}\left(\alpha_{1}(x)\right)$. In general, starting from some $x$ in $S$, one has

$$
X_{n+1}(x)=\alpha_{n+1}\left(X_{n}(x)\right),
$$

where the maps $\left(\alpha_{n}\right)$ are independent with the common distribution $Q$. The initial point $x$ can also be chosen (independently of $\left(\alpha_{n}\right)$ ) as a random variable $X_{0}$. The sequence $X_{n}$ of states obtained in this manner is a Markov process and has been of particular interest in developing stochastic dynamic models in many disciplines. With specific assumptions on the structure of $S$ and $\Gamma$ it has been possible to derive strong results on the asymptotic behavior of $X_{n}$.

Random dynamical systems have been particularly useful for modeling long run evolution of economic systems subject to exogenous random shocks. The framework (1.1) can be interpreted as a descriptive model; but, one may also start with a discounted (stochastic) dynamic programming problem, and directly arrive at a stationary optimal policy function, which together with the exogenously given law of transition describes the optimal evolution of the states in the form (1.1). Of particular significance are results on the "inverse optimal problem under uncertainty" due to Mitra (1998) and Montrucchio and Privileggi (1999) which assert that a very broad class of random systems (1.1) can be so interpreted. 
The literature exploring (1.1) is already vast and growing. Given the space limitations, this review is primarily restricted to the case when $S$ is an interval (non-degenerate) in $R$, or a closed (nonempty) subset of $R^{\ell}$, and $\Gamma$ is a family of monotone maps from $S$ into $S$. Some extensions to more general framework and applications are also outlined. Here I touch upon a few of the issues and provide some references to definitive treatments.

(i) The existence, uniqueness and global stability of a steady state (an invariant distribution) of random dynamical systems: Significant progress has been achieved when the laws of motion satisfy either some "splitting" or "contraction"conditions (see, e.g., Dubins and Freedman (1966), Diaconis and Freedman (1999) Bhattacharya and Majumdar (1999, 2001) and the review in B-M (2007, Chapter 3)). An awkward problem involving the existence question is worth-noting. Consider $S=[0,1]$ or $S=R_{+}$and assume that $\gamma(0)=0$ for all $\gamma \in \Gamma$. This is a natural property of a law of motion in many population or economic models (viewed as a production function, $\gamma(0)=0$ means that zero input leads to zero output). The point mass at 0 (the measure $\delta_{0}$ ) is obviously an invariant distribution. The challenge, then, is to find an invariant distribution with support in $(0,1)$.

(ii) The nature of the invariant distribution. Suppose, for concreteness, that $S$ is an interval, and $F$ is the distribution function on $R$ of the unique invariant measure. Invoking a standard decomposition property (see Loeve (1960, p. 130, 196), let (i) $F_{d}$ be the step part (a step function); (ii) $F_{a c}$ be the absolutely continuous part (with respect to the Lebesgue measure) and (iii) $F_{S}$ be the singular part of $F$.

As a first step one would like to know whether (i) $F$ is continuous ( $F_{d} \equiv 0$ ) or whether (ii) $F$ is absolutely continuous or whether (iii) $F$ is singular. At the next step, one would like to ask questions of comparative statics: how does $F$ (or the components (i) - (iii)) change if a parameter in the model is allowed to change? Finally, one would like to compute (or approximate) $F$ but that typically requires more structure on the model.

All the questions are elusive. Take the standard approach of describing a Markov process with state space $S=R$, and a transition function $p(x, A)$. If for each $x \in S, p(x,$.$) is absolutely$ 
continuous with respect to the Lebesgue measure, then if $\pi$ is invariant under $p(x, A), \pi$ is also absolutely continuous with respect to the Lebesgue measure [see B-M (2007, Proposition 5.2 of Chapter 5]. This result is to be contrasted with those in Section 6.2.

A study of (i.i.d) random iteration of quadratic maps $(S=[0,1], \Gamma=\{f: f(x)=\theta x(1-x)$, $0 \leq \theta \leq 4\}, Q$ with a two point support) was initiated by Bhattacharya and Rao (1993). The subsequent literature offers interesting examples on applications of splitting and open questions. For a review of results when $\Gamma$ is the quadratic family (the typical $\gamma(x)=\theta x(1-x)$ does not satisfy the monotonicity property that is central here but does have 'piecewise monotonicity' which has often been used to invoke the splitting conditions: see Athreya and Bhattacharya (2000); further extensions are in Athreya (2004)).

The processes considered in this article particularly when $\Gamma$ is finite are not in general Harris irreducible (see, e.g., Orey (1971) for a definition of Harris irreducibility). Therefore, the standard techniques used for the study of irreducible Markov processes in the literature are not applicable to many of the cases reviewed. This point was explored in detail in Ellner (1984) who concluded that "it is surprising and unfortunate that the large classical theory based on compactness and/or irreducibility conditions generally give little information about (1.1) as a population model." The reader interested in this issue is referred to Ellner (1984, Section 5).

(iii) Applications of the theoretical results to a few topics:

(a) turnpike theorems in the literature on descriptive and optimal growth under uncertainty: when each admissible law of motion is monotone increasing, and satisfies the appropriate Inada-type 'end point' condition, Theorem 4.1 can be applied directly.

(b) estimation of the invariant distribution: as noted above, an important implication of the "splitting theorems" is an estimate of the speed of convergence. This estimate is used in Section 5 to prove a result on $\sqrt{n}$-consistency of the sample mean as an estimator of the expected long run equilibrium value (i.e., the value of the state variable with respect to the invariant distribution). 


\section{Random Dynamical Systems}

We consider random dynamical systems. Let $S$ be a metric space and $\mathcal{S}$ be the Borel $\sigma$-field of $S$. Endow $\Gamma$ with a $\sigma$-field $\Sigma$ such that the map $(\gamma, x) \rightarrow(\gamma(x))$ on $(\Gamma \times S, \Sigma \otimes \mathcal{S}$ into $(S, \mathcal{S})$ is measurable. Let $Q$ be a probability measure on $(\Gamma, \Sigma)$. On some probability space $(\Omega, \digamma, P)$ let $\left(\alpha_{n}\right)_{n=1}^{\infty}$ be a sequence of independent random functions from $\Gamma$ with a common distribution $Q$.

For a given random variable $X_{0}$ (with values in $S$ ), independent of the sequence $\left(\alpha_{n}\right)_{n=1}^{\infty}$, define

$$
\begin{gathered}
X_{1} \equiv \alpha_{1}\left(X_{0}\right) \equiv \alpha_{1} X_{0} \\
X_{n+1}=\alpha_{n+1}\left(X_{n}\right) \equiv \alpha_{n+1} \alpha_{n} \ldots \alpha_{1} X_{0}
\end{gathered}
$$

We write $X_{n}(x)$ for the case $X_{0}=x$; to simplify notation we write $X_{n}=\alpha_{n} \ldots \alpha_{1} X_{0}$ for the more general (random) $X_{0}$. Then $X_{n}$ is a Markov process with the stationary transition probability $p(x, d y)$ given as follows: for $x \in S, C \in \mathcal{S}$,

$$
p(x, C)=Q(\{\gamma \in \Gamma: \gamma(x) \in C\})
$$

The stationary transition probability $p(x, d y)$ is said to be weakly continuous or to have the Feller property if for any sequence $x_{n}$ converging to $x$, the sequence of probability measures $p\left(x_{n}, \cdot\right)$ converges weakly to $p(x, \cdot)$. One can show that if $\Gamma$ consists of a family of continuous maps, $p(x, d y)$ has the Feller property.

\section{Evolution}

To study the evolution of the process $(2.2)$, it is convenient to define the map $T^{*}$ [on the space $M(S)$ of all finite signed measures on $(S, \mathcal{S})]$ by

$$
T^{*} \mu(C)=\int_{S} p(x, C) \mu(d x)=\int_{\Gamma} \mu\left(\gamma^{-1} C\right) Q(d \gamma), \quad \mu \in M(S)
$$


Let $\mathcal{P}(S)$ be the set of all probability measures on $(S, \mathcal{S})$. An element $\pi$ of $\mathcal{P}(S)$ is invariant for $p(x, d y)$ (or for the Markov process $X_{n}$ ) if it is a fixed point of $T^{*}$, i.e.,

$$
\pi \text { is invariant iff } \quad T^{*} \pi=\pi
$$

Now write $p^{(n)}(x, d y)$ for the $n$-step transition probability with $p^{(1)} \equiv p(x, d y)$. Then $p^{(n)}(x, d y)$ is the distribution of $\alpha_{n} \ldots . . \alpha_{1} x$. Define $T^{* n}$ as the $n$-th iterate of $T^{*}$ :

$$
T^{* n} \mu=T^{*(n-1)}\left(T^{*} \mu\right)(n \geq 2), T^{* 1}=T^{*}, T^{* 0}=\text { Identity }
$$

Then for any $C \in \mathcal{S}$,

$$
\left(T^{* n} \mu\right)(C)=\int_{S} p^{(n)}(x, C) \mu(d x),
$$

so that $T^{* n} \mu$ is the distribution of $X_{n}$ when $X_{0}$ has distribution $\mu$. To express $T^{* n}$ in terms of the common distribution $Q$ of the i.i.d. maps $\left(\alpha_{n}\right)$, let $\Gamma^{n}$ denote the usual Cartesian product $\Gamma \times \Gamma \times \ldots \times \Gamma(n$ terms $)$, and let $Q^{n}$ be the product probability $Q \times Q \times \ldots \times Q$ on $\left(\Gamma^{n}, \mathcal{S}^{\otimes n}\right)$ where $\mathcal{S}^{\otimes n}$ is the product $\sigma$-field on $\Gamma^{n}$. Thus $Q^{n}$ is the (joint) distribution of $\boldsymbol{\alpha}=\left(\alpha_{1}, \alpha_{2}, \ldots, \alpha_{n}\right)$. For $\gamma=\left(\gamma_{1}, \gamma_{2}, \ldots, \gamma_{n}\right) \epsilon \Gamma^{n}$ let $\tilde{\gamma}$ denote the composition

$$
\tilde{\gamma}:=\gamma_{n} \gamma_{n-1} \cdots \gamma_{1}
$$

We suppress the dependence of $\tilde{\gamma}$ on $n$ for notational simplicity. Then, since $T^{* n} \mu$ is the distribution of $X_{n}=\alpha_{n} \ldots \alpha_{1} X_{0}$, one has $\left(T^{* n} \mu\right)(A)=\operatorname{Prob}\left(X_{0} \epsilon \tilde{\boldsymbol{\alpha}}^{-1} A\right)$, where $\tilde{\boldsymbol{\alpha}}=\alpha_{n} \alpha_{n-1} \ldots \alpha_{1}$. Therefore, by the independence of $\tilde{\boldsymbol{\alpha}}$ and $X_{0}$,

$$
\left(T^{* n} \mu\right)(A)=\int_{\Gamma^{n}} \mu\left(\tilde{\gamma}^{-1} A\right) Q^{n}(d \gamma) \quad(A \in \mathcal{S}, \mu \in \mathcal{P}(S))
$$

Finally, we come to the definition of stability. A Markov process $X_{n}$ is stable in distribution if there is a unique invariant probability measure $\pi$ such that $X_{n}(x)$ converges weakly (or, in 
distribution) to $\pi$ irrespective of the initial state $x$, i.e., if $p^{(n)}(x, d y)$ converges weakly to the same probability measure $\pi$ for all $x$.

In what follows, if $g$ is a bounded $\mathcal{S}$-measurable real valued function on $S$, we write

$$
T g(x)=\int_{S} g(y) p(x, d y)
$$

\section{$4 \quad$ Splitting}

If $S$ is a (nonempty) compact metric space and $\Gamma$ consists of a family of continuous functions

from $S$ into $S$, then a fixed point argument ensures that there is an invariant probability measure $\pi^{*}$. However, when $\Gamma$ consists of monotone maps on a suitable subset $S$ of $R^{\ell}$ (into $S$ ), stronger results on uniqueness and stability can be derived by using a 'splitting' condition, first studied by Dubins and Freedman (1966).

\subsection{Splitting and Monotone Maps}

Let $S$ be a nondegenerate interval (finite or infinite, closed, semiclosed, or open) and $\Gamma$ a set of monotone maps from $S$ into $S$; i.e., each element of $\Gamma$ is either a nondecreasing function on $S$ or a nonincreasing function.

We assume the following splitting condition:

(H) There exist $z_{0} \in S, \tilde{\chi}>0$ and a positive $N$ such that

(1) $\quad P\left(\alpha_{N} \alpha_{N-1} \ldots \alpha_{1} x \leq z_{0} \forall x \in S\right) \geq \tilde{\chi}$

(2) $\quad P\left(\alpha_{N} \alpha_{N-1} \ldots \alpha_{1} x \geq z_{0} \forall x \in S\right) \geq \tilde{\chi}$.

Note that conditions (1) and (2) in ( $\mathbf{H})$ may be expressed, respectively, as

$$
Q^{N}\left(\left\{\gamma \in \Gamma^{N}: \tilde{\gamma}^{-1}\left[x \in S: x \leq z_{0}\right]=S\right\}\right) \geq \tilde{\chi}
$$


and

$$
Q^{N}\left(\left\{\gamma \in \Gamma^{N}: \tilde{\gamma}^{-1}\left[x \in S: x \geq z_{0}\right]=S\right\}\right) \geq \tilde{\chi}
$$

Recall that $\tilde{\gamma}=\gamma_{N} \gamma_{N-1} \ldots \gamma_{1}$.

Denote by $d_{K}(\mu, \nu)$ the Kolmogorov distance on $\mathcal{P}(S)$. That is, if $F_{\mu}, F_{\nu}$ denote the distribution functions (d.f.) of $\mu$ and $\nu$, respectively, then

$$
\begin{aligned}
d_{K}(\mu, \nu) & \left.: \quad \sup _{x \in R} \mid \mu((-\infty, x] \cap S)-\nu(-\infty, x] \cap S\right) \mid \\
\equiv & \sup _{x \in R}\left|F_{\mu}(x)-F_{\nu}(x)\right|, \mu, \nu \in \mathcal{P}((S)) .
\end{aligned}
$$

Remark 4.1 First, it should be noted that convergence in the distance $d_{K}$ on $\mathcal{P}(S)$ implies weak convergence in $\mathcal{P}(S)$. Secondly, $\left(\mathcal{P}(S), d_{K}\right)$ is a complete metric space. (See B-M $[2007$, Theorems 5.1 and C11.2(d) of Chapter 2]).

Theorem 4.1 Assume that the splitting condition $(\boldsymbol{H})$ holds.

Then

(a) the distribution $T^{* n} \mu$ of $X_{n}:=\alpha_{n} \ldots \alpha_{1} X_{0}$ converges to a probability measure $\pi$ on $S$ in the Kolmogorov distance $d_{K}$ irrespective of $X_{0}$. Indeed,

$$
d_{K}\left(T^{* n} \mu, \pi\right) \leq(1-\tilde{\chi})^{[n / N]} \forall \mu \in \mathcal{P}(S)
$$

where $[y]$ denotes the integer part of $y$.

(b) $\pi$ in (a) is the unique invariant probability of the Markov process $X_{n}$.

Main Steps. Careful calculations using the splitting condition and monotonicity lead to (see B-M (2007, Chapter 3, Theorem 5.1):

$$
d_{K}\left(T^{*} \mu, T^{*} \nu\right) \leq d_{K}(\mu, \nu)
$$


and

$$
d_{K}\left(T^{* N} \mu, T^{* N} \nu\right) \leq(1-\tilde{\chi}) d_{K}(\mu, \nu) \quad(\mu, \nu \in \mathcal{P}(S))
$$

That is, $T^{* N}$ is a uniformly strict contraction and $T^{*}$ is a contraction. As a consequence, $\forall_{n}>N$, one has

$$
\begin{aligned}
d_{K}\left(T^{* n} \mu, T^{* n} \nu\right) & =d_{K}\left(T^{* N}\left(T^{*(n-N)} \mu\right), T^{* N}\left(T^{*(n-N)} \nu\right)\right) \\
& \leq(1-\tilde{\chi}) d_{K}\left(T^{*(n-N)} \mu, T^{*(n-N)} \nu\right) \leq \ldots \\
& \leq(1-\tilde{\chi})^{[n / N]} d_{K}\left(T^{*(n-[n / N] N)} \mu, T^{*(n-[n / N] N)} \nu\right) \\
& \leq(1-\tilde{\chi})^{[n / N]} d_{K}(\mu, \nu) .
\end{aligned}
$$

Now, by appealing to the contraction mapping theorem, $T^{* N}$ has a unique fixed point $\pi$ in $\mathcal{P}(S)$, and $T^{* N}\left(T^{*} \pi\right)=T^{*}\left(T^{* N} \pi\right)=T^{*} \pi$. Hence $T^{*} \pi$ is also a fixed point of $T^{* N}$. By uniqueness $T^{*} \pi=\pi$. Hence, $\pi$ is a fixed point of $T^{*}$. Any fixed point of $T^{*}$ is a fixed point of $T^{* N}$. Hence $\pi$ is the unique fixed point of $T^{*}$. Now take $\nu=\pi$ in (4.7) to get the desired relation (4.4).

The following remarks clarify the role of the splitting condition.

Remark 4.2 Let $S=[a, b]$ and $\alpha_{n}(n \geq 1)$ a sequence of i.i.d. continuous nondecreasing maps on $S$ into $S$. Suppose that $\pi$ is the unique invariant distribution of the Markov process. If $\pi$ is not degenerate, then the splitting condition holds [Dubins and Freedman (1966, Theorem 5.17); for relaxing continuity, see B-M (2007, Lemma CS.2 of Chapter 3)].

Remark 4.3 Suppose that $\alpha_{n}$ are strictly monotone a.s. Then if the initial distribution $\mu$ is nonatomic (i.e., $\mu(\{x\})=0 \forall x$ or, equivalently the d.f. of $\mu$ is continuous), $\mu o \gamma^{-1}$ is nonatomic $\forall \gamma \in \Gamma$ (outside a set of zero Q-probability). It follows that if $X_{0}$ has a continuous d.f., then so has $X_{1}$ and in turn $X_{2}$ has a continuous d.f., and so on. Since, by Theorem 4.1, this sequence of continuous d.f.s (of $X_{n}(n \geq 1)$ ) converges uniformly to the d.f. of $\pi$, the latter is continuous. Thus $\pi$ is nonatomic if $\alpha_{n}$ are strictly monotone a.s.

Example 4.1 Let $S=[0,1]$ and $\Gamma$ be a family of monotone nondecreasing functions from 
$S$ into $S$. As before, for any $z \in S$, let

$$
X_{n}(z)=\alpha_{n} \ldots \alpha_{1} z
$$

One can verify the following two results:

[R.1] $\quad P\left[X_{n}(0) \leq x\right]$ is nonincreasing in $n$ and converges for each $x \in S$.

[R.2] $\quad P\left[X_{n}(1) \leq x\right]$ is nondecreasing in $n$ and converges for each $x \in S$.

Write

$$
\begin{aligned}
& F_{0}(x) \equiv \lim _{n \longrightarrow \infty} P\left(X_{n}(0) \leq x\right) . \\
& F_{1}(x) \equiv \lim _{n \longrightarrow \infty} P\left(X_{n}(1) \leq x\right) .
\end{aligned}
$$

Note that $F_{1}(x) \leq F_{0}(x)$ for all $x$. Consider the case when $\Gamma \equiv\{f\}$, where

$$
f(x)=\left\{\begin{array}{l}
\frac{1}{4}+\frac{x}{4} \text { if } 0 \leq x<\frac{1}{3} \\
\frac{1}{3}+\frac{x}{3} \text { if } \frac{1}{3} \leq x \leq \frac{2}{3} \\
\frac{1}{3}+\frac{x}{2} \text { if } \frac{2}{3}<x \leq 1
\end{array}\right.
$$

Verify that $f$ is a monotone increasing map from $S$ into $S$, but $f$ is not continuous. One can calculate that

$$
F_{0}(x)=\left\{\begin{array}{l}
0 \text { if } 0 \leq x<\frac{1}{3}, \\
1 \text { if } \frac{1}{3} \leq x \leq 1 .
\end{array} \quad F_{1}(x)=\left\{\begin{array}{l}
0 \text { if } 0 \leq x<\frac{2}{3} \\
1 \text { if } \frac{2}{3} \leq x \leq 1 .
\end{array}\right.\right.
$$

Neither $F_{0}$ nor $F_{1}$ is a stationary distribution function.

Example 4.2 Let $S=[0,1]$ and $\Gamma=\left\{f_{1}, f_{2}\right\}$. In each period $f_{1}$ is chosen with probability $\frac{1}{2}$. $f_{1}$ is the function $f$ defined in Example 4.1, and $f_{2}(x)=\frac{1}{3}+\frac{x}{3}$, for $x \in S$.

Then

$$
F_{0}(x)=F_{1}(x)= \begin{cases}0 & \text { if } 0 \leq x<\frac{1}{2} \\ 1 & \text { if } \frac{1}{2} \leq x \leq 1\end{cases}
$$

and $F_{0}(x)$ is the unique stationary distribution. Note that $f_{1}\left(\frac{1}{2}\right)=f_{2}\left(\frac{1}{2}\right)=\frac{1}{2}$, i.e., $f_{1}$ and $f_{2}$ 
have a common fixed point. Examples 4.1 and 4.2 are taken from Yahav (1975).

We now turn to the case where the state space is a subset of $R^{\ell}(\ell \geq 1)$ satisfying the following assumption:

(A.1) $\quad S$ is a closed subset of $R^{\ell}$.

Let $\Gamma$ be a set of monotone maps $\gamma$ on $S$ into $S$, under the partial order: $\mathbf{x} \leq \mathbf{y}$ if $x_{j} \leq y_{j}$ for $1 \leq j \leq \ell ; \mathbf{x}=\left(x_{1}, \ldots, x_{\ell}\right), \mathbf{y}=\left(y_{1}, y_{2}, \ldots, y_{\ell}\right) \epsilon R^{\ell}$ (or $\left.S\right)$. That is, either $\gamma$ is monotone increasing: $\gamma(\mathbf{x}) \leq \gamma(\mathbf{y})$ if $\mathbf{x} \leq \mathbf{y}$, or $\gamma$ is monotone decreasing: $\gamma(\mathbf{y}) \leq \gamma(\mathbf{x})$ if $\mathbf{x} \leq \mathbf{y} ; \mathbf{x}, \mathbf{y} \epsilon S$.

On the space $\mathcal{P}(S)$, define, for each $a>0$, the metric

$$
d_{a}(\mu, \nu)=\sup _{g \in \mathcal{G}_{a}}\left|\int g d \mu-\int g d \nu\right|,(\mu, \nu \in \mathcal{P}(S))
$$

where $\mathcal{G}_{a}$ is the class of all Borel measurable monotone (increasing or decreasing) functions $g$ on $S$ into $[0, a]$. The following result is due to Chakraborty and Rao (1998), who derived a number of interesting results on the metric space $\left(\mathcal{P}(S), d_{a}\right)$. One can show that convergence in the metric $d_{a}$ implies weak convergence if (A.1) holds (see B-M (2007, pp. 287-288)).

Lemma 4.1 Under the hypothesis $(A .1),\left(\mathcal{P}(S), d_{a}\right)$ is a complete metric space.

Consider the following splitting condition $\left(\mathbf{H}^{\prime}\right)$. To state it, let $\tilde{\gamma}$ be as in (3.5), but with $n=N: \tilde{\gamma}=\gamma_{N} \gamma_{N-1} \ldots \gamma_{1}$ for $\gamma=\left(\gamma_{1}, \gamma_{2}, \ldots, \gamma_{N}\right) \epsilon \Gamma^{N}$

$\left(\mathrm{H}^{\prime}\right) \quad$ There exist $F_{i} \epsilon \sum^{\otimes N}(i=1,2)$ for some $N \geq 1$, such that

(i) $\quad \delta_{i} \equiv Q^{N}\left(F_{i}\right)>0(i=1,2)$, and

(ii) for some $\mathbf{x}_{\mathbf{0}} \epsilon S$, one has

$$
\begin{aligned}
& \tilde{\gamma}(\mathbf{x}) \leq \mathbf{x}_{0} \forall \mathbf{x} \epsilon S, \quad \forall \gamma \in F_{1}, \\
& \tilde{\gamma}(\mathbf{x}) \geq \mathbf{x}_{0} \quad \forall \mathbf{x} \epsilon S, \quad \forall \gamma \in F_{2},
\end{aligned}
$$

Also, assume that the set $H_{+}=\left\{\gamma \epsilon \Gamma^{N}: \tilde{\gamma}\right.$ is monotone increasing $\} \epsilon \sum^{\otimes N}$. 
Theorem 4.2 Let $\left\{\alpha_{n}: n \geq 1\right\}$ be a sequence of i.i.d. measurable monotone maps with a common distribution $Q$. Assume (A.1) and $\left(\boldsymbol{H}^{\prime}\right)$ hold. Then there exists a unique invariant probability measure for the Markov process (2.1) and

$$
\sup _{\mathbf{x} \in S} d_{1}\left(p^{(n)}(\mathbf{x}, .), \pi\right) \leq(1-\delta)^{\left[\frac{n}{N}\right]}(n \geq 1)
$$

where $\delta=\min \left\{\delta_{1}, \delta_{2}\right\}$, and $\left[\frac{n}{N}\right]$ is the integer part of $\frac{n}{N}$.

Proof. The proof uses Lemma 4.1 and is spelled out in Bhattacharya and Majumdar (2007). As in the case of Theorem 4.1, we prove:

Step 1. $T^{* N}$ is a uniformly strict contraction on $\left(\mathcal{P}(S), d_{1}\right)$

In other words,

$$
d_{1}\left(T^{* N} \mu, T^{* N} \nu\right) \leq(1-\delta) d_{1}(\mu, \nu), \forall \mu, \nu \in \wp(S) .
$$

Now, Step 2. Apply the Contraction Mapping Theorem.

For earlier related results see Bhattacharya and Lee (1988).

\subsection{An Extension and Some Applications}

An extension of Theorems 4.1 - 4.2 [proved in Bhattacharya and Majumdar (1997)] is useful for applications. Recall that $\mathcal{S}$ is the Borel $\sigma$-field of the state space $S$. Let $\mathcal{A} \subset \mathcal{S}$, define

$$
d(\mu, \nu):=\sup _{A \in \mathcal{A}}|\mu(A)-\nu(A)| \quad(\mu, \nu \in \mathcal{P}(S)) .
$$

(1) Consider the following hypothesis $\left(\mathbf{H}_{1}\right)$ :

$$
(\mathcal{P}(S), d) \text { is a complete metric space; }
$$


(2) there exists a positive integer $N$ such that for all $\gamma \in \Gamma^{N}$, one has

$$
d\left(\mu \tilde{\gamma}^{-1}, \nu \tilde{\gamma}^{-1}\right) \leq d(\mu, \nu)(\mu, \nu \in \mathcal{P}(S))
$$

(3) there exists $\delta>0$ such that $\forall A \in \mathcal{A}$, and with $N$ as in (2), one has

$$
P\left(\tilde{\boldsymbol{\alpha}}^{-1}(A)=S \text { or } \phi\right) \geq \delta>0
$$

Theorem 4.3 Assume the hypothesis $\left(\mathbf{H}_{1}\right)$. Then there exists a unique invariant probability $\pi$ for the Markov process $X_{n}:=\alpha_{n} \ldots \alpha_{1} X_{0}$, where $X_{0}$ is independent of $\left\{\alpha_{n}:=n \geq 1\right\}$. Also, one has

$$
d\left(T^{* n} \mu, \pi\right) \leq(1-\delta)^{[n / N]} \quad(\mu \epsilon \mathcal{P}(S))
$$

where $T^{* n} \mu$ is the distribution of $X_{n}$ when $X_{0}$ has distribution $\mu$, and $[n / N]$ is the integer part of $n / N$.

Remark 4.4 For applications of Theorem 4.3 to derive a Doeblin-type convergence theorem, and to the study of non-linear autoregressive processes see B-M (2007).

\subsection{Extinction and Growth}

Some light has been thrown on the possibilities of growth and extinction. To review these results (see Ellner (1984) for proofs and other related results), let us assume that $S=[0, \infty)$, and $\Gamma$ consists of a family of maps $f: S \rightarrow S$ satisfying

C.1 $f(x)$ is continuously differentiable and strictly increasing on $[0, \infty)$

C.2 $\frac{d}{d x}\left[x^{-1} f(x)\right]<0$ for $x>0$ (concavity)

C.3 There is some $K>0$ such that $f(K)<K$ for all $f \in \Gamma$ (note that $K$ is independent of $f$ )

Then we have the following:

Theorem 4.4 Suppose $0<X_{0}<K$ with probability one. Then: 
a) $\quad X_{n}$ converges in distribution to a stationary distribution;

b) The stationary distribution is independent of $X_{0}$ and its df has $F\left(0^{+}\right)=0$ or $1\left[F\left(0^{+}\right)=\right.$ 1 means that $X_{n} \stackrel{w}{\rightarrow} 0$, which is extinction of the population].

It is often useful to study the non-linear stochastic difference equation written in formally as:

$$
X_{n+1}=f\left(X_{n}, \theta_{n+1}\right)
$$

where $\left(\theta_{n}\right)$ is a sequence of independent, identically distributed random variables taking values in a (nonempty) finite set $\mathbb{A} \subset R_{++}$. Here $f: R_{+} \times \mathbb{A} \rightarrow R_{+}$satisfies, for each $\theta \in \mathbb{A}$ the conditions (C.1) - (C.2). Write $R(x, \theta)=x^{-1} f(x, \theta)$ for $x>0$.

For each $\theta \in \mathbb{A}$, let

$$
R(0, \theta)=\lim _{x \rightarrow 0^{+}} R(x, \theta)
$$

and

$$
R(\infty, \theta)=\lim _{x \rightarrow \infty} R(x, \theta) \equiv f^{\prime}(x, \theta)
$$

Define the growth rates

$$
v_{0}=E[(\log R(0, \theta)]
$$

and

$$
v_{\infty}=E[(\log R(\infty, \theta)]
$$

By C.2 $v_{0}$ and $v_{\infty}$ are well-defined.

Theorem 4.5 Under assumptions C.1 - C.2 and $0<X_{0}<\infty$ with probability one,

a) if $v_{0} \leq 0, X_{n} \rightarrow 0$ with probability one

b) if $v_{\infty} \geq 0, X_{n} \rightarrow \infty$ with probability one

c) if $v_{0}>0, v_{\infty}<0, X_{n}$ converges weakly (independently of the distribution of $X_{0}$ ) to a distribution with support in $(0, \infty)$. 


\section{Invariant Distributions: Computation and Estimation}

The problem of deriving analytical properties of invariant distributions has turned out to be difficult and elusive. In this section we provide an example of a class of Markov processes in which the unique invariant distribution can be completely identified.

Let $Z_{1}, Z_{2} \ldots$, be a sequence of non-negative i.i.d. random variables. Consider the Markov Chain $\left\{X_{n}: n=0,1,2 \ldots\right\}$ on the state space $S=R_{++}$defined by

$$
X_{n+1}=Z_{n+1}+\left[1 / X_{n}\right] \quad n \geq 0
$$

where $X_{0}$ is a strictly positive random variable independent of the sequence $\left\{Z_{i}\right\}$. We first summarize the dynamic behavior of the sequence $\left\{X_{n}\right\}$.

Theorem 5.1 Assume that $\left\{Z_{i}\right\}$ are non-degenerate. Then the Markov chain $\left\{X_{n}, n=0,1\right\}$ on $S=R_{++}$has a unique invariant probability $\pi$, and $d_{k}\left(T^{* n} \mu, \pi\right)$ converges to zero exponentially fast, irrespective of the initial distribution $\mu$ and the invariant probability $\pi$ is non-atomic.

Proof. The main step in the proof is to represent $X_{n}$ as

$$
X_{n}=\alpha_{n} \cdot \alpha_{n-1} \ldots \alpha_{1}\left(X_{0}\right)
$$

where $\alpha_{n}(x)=Z_{n}+1 / x, n \geq 1$. The maps $\alpha_{n}$ are monotone decreasing on $S$. The splitting condition can also be verified (see Goswami 2004, Theorem 4.1). Hence Theorem 3.1 can be applied directly.

Suppose that the common distribution of $Z_{i}$ is a Gamma distribution. Recall that the Gamma distribution with parameters $\lambda>0$ and $a>0$ is the distribution on $R_{++}$given by the density function

$$
\gamma_{\lambda, a}(z)=\left\{\begin{array}{lr}
\frac{a^{\lambda}}{\Gamma(\lambda)} z^{\lambda-1} e^{-a z} & \text { if } z \epsilon R_{++} \\
0 & \text { otherwise }
\end{array}\right.
$$


where $\Gamma(\cdot)$ is the gamma function:

$$
\Gamma(\beta)=\int_{0}^{\infty} x^{\beta-1} e^{-x} d x
$$

Theorem 5.2 Suppose that the common distribution of the i.i.d. sequence $\left\{Z_{i}\right\}$ is a Gamma distribution with parameters $\lambda$ and $a$. Then the invariant probability $\pi$ on $(0, \infty)$ is absolutely continuous with density function

$$
g_{\lambda, a}(x)=\left(2 K_{\lambda}(2 a)\right)^{-1} x^{\lambda-1} e^{-a\left(x+\frac{1}{x}\right)}, x \epsilon R_{++}
$$

where $K_{\lambda}(\cdot)$ denotes the Bessel function, i.e., $K_{\lambda}(z)=\frac{1}{2} \int_{0}^{\infty} x^{\lambda-1} e^{-\frac{1}{2} z\left(x+\frac{1}{x}\right)} d x$.

Another interesting example corresponds to Bernoulli $Z_{i}: \mathcal{P}\left(Z_{i}=0\right)=p, \mathcal{P}\left(Z_{i}=1\right)=$ $1-p(0<p<1)$. In this case the unique invariant distribution $\pi$ is singular with respect to Lebesgue measure, and has full support on $S=(0, \infty)$. An explicit computation of the distribution function of $\pi$, involving the classical continued fraction expansion of the argument, may be found in Goswami (2002, Theorem 5.2).

\subsection{An Estimation Problem}

Consider a Markov chain $X_{n}$ with a unique stationary distribution $\pi$. Some of the celebrated results on ergodicity and the strong law of large numbers hold for $\pi$-almost every initial condition. However, even with $[0,1]$ as the state space, the invariant distribution $\pi$ may be hard to compute explicitly when the laws of motion are allowed to be non-linear, and its support may be difficult to characterize or may be a set of zero Lebesgue measure. Moreover, in many economic models, the initial condition may be historically given, and there may be little justification in assuming that it belongs to the support of $\pi$.

Consider, then, a random dynamical system with state space $[c, d]$ (without loss of generality for what follows choose $c>0$ ). Assume $\Gamma$ consists of a family of monotone maps from $S$ with $S$, 
and the splitting condition $(\mathbf{H})$ hold. The process starts with a given $x$. There is, by Theorem 4.1, a unique invariant distribution $\pi$ of the random dynamical system, and (4.4) holds. Suppose we want to estimate the equilibrium mean $\int_{S} y \pi(d y)$ by sample means $\frac{1}{n} \sum_{j=0}^{n-1} X_{j}$. We say that the estimator $\frac{1}{n} \sum_{j=0} X_{j}$ is $\sqrt{n}$-consistent if

$$
\frac{1}{n} \sum_{j=0}^{n-1} X_{j}=\int y \pi(d y)+O_{P}\left(n^{-1 / 2}\right)
$$

where $O_{p}\left(n^{-1 / 2}\right)$ is a random sequence $\varepsilon_{n}$ such that $\left|\varepsilon_{n} \cdot n^{1 / 2}\right|$ is bounded in probability. Thus, if the estimator is $\sqrt{n}$-consistent, the fluctuations of the empirical (or sample-) mean around the equilibrium mean is $O_{p}\left(n^{-1 / 2}\right)$. We can establish (5.1) by using (4.4). One can show that (see Bhattacharya, and Majumdar [2001, pp. 217-219]) if

$$
f(z)=z-\int y \pi(d y)
$$

then

$$
\sup _{x} \sum_{n=m+1}^{\infty}\left|T^{n} f(x)\right| \leq(d-c) \sum_{n=m+1}^{\infty}(1-\delta)^{[n / N]} \rightarrow 0 \quad \text { as } m \rightarrow \infty
$$

Hence, $g=-\sum_{n=0}^{\infty} T^{N} f$ [where $T^{0}$ is the identity operator $\left.I\right]$ is well-defined, and $g$, and $T g$ are bounded functions. Also, $(T-I) g=-\sum_{n=1}^{\infty} T^{n} f+\sum_{n=0}^{\infty} T^{N} f \equiv f$. Hence,

$$
\begin{aligned}
\sum_{j=0}^{n-1} f\left(X_{j}\right) & =\sum_{j=0}^{n-1}(T-I) g\left(X_{j}\right) \\
& =\sum_{j=0}^{n-1}\left((T g)\left(X_{j}\right)-g\left(X_{j}\right)\right) \\
& =\sum_{j=1}^{n}\left[(T g)\left(X_{j-1}\right)-g\left(X_{j}\right)\right]+g\left(X_{n}\right)-g\left(X_{0}\right)
\end{aligned}
$$

By the Markov property and the definition of $\mathrm{Tg}$ it follows that

$$
E\left((T g)\left(X_{j-1}\right)-g\left(X_{j}\right) \mid \mathcal{F}_{j-1}\right)=0
$$


where $\mathcal{F}_{r}$ is the $\sigma$-field generated by $\left\{X_{j}: 0 \leq j \leq r\right\}$. Hence, $(T g)\left(X_{j-1}\right)-g\left(X_{j}\right)(j \geq 1)$ is a martingale difference sequence, and are uncorrelated, so that

$$
E\left[\sum_{j=1}^{k}\left(T g\left(X_{j-1}\right)-g\left(X_{j}\right)\right)\right]^{2}=\sum_{j=1}^{n} E\left((T g)\left(X_{j-1}\right)-g\left(X_{j}\right)\right)^{2}
$$

Given the boundedness of $g$ and $T g$, the right side is bounded by $n . \alpha$ for some constant $\alpha$. It follows that

$$
\frac{1}{n} E\left(\sum_{j=0}^{n-1} f\left(X_{j}\right)\right)^{2} \leq \eta^{\prime} \quad \text { for all } n
$$

where $\eta^{\prime}$ is a constant that does not depend on $X_{0}$. Thus,

$$
E\left(\frac{1}{n} \sum_{j=0}^{n-1} X_{j}-\int y \pi(d y)\right)^{2} \leq \eta^{\prime} / n
$$

which implies,

$$
\frac{1}{n} \sum_{j=0}^{n-1} X_{j}=\int y \pi(d y)+0_{p}\left(n^{-1 / 2}\right)
$$

For other examples of $\sqrt{n}$-consistent estimation, see Athreya and Majumdar (2002) [and B-M (2008, Chapter 5)].

\section{Growth Under Uncertainty}

\subsection{A Stochastic Stability Theorem in a Descriptive Model}

Models of descriptive as well as optimal growth under uncertainty have led to random dynamical systems that are stable in distribution. We look at a "canonical" example and show how Theorem 4.1 can be applied. We begin with a descriptive growth model and follow it up with an optimization problem.

As a matter of notation, for any function $h$ on $S$ into $S$, we write $h^{(n)}$ for the $n$th iterate of 
$h$. Think of ' $x$ ' as per capital output of an economy.

Let $S=R_{+}$; and $\Gamma=\left\{F_{1}, F_{2}, \ldots, F_{i}, \ldots, F_{N}\right\}$ where the distinct laws of motion $F_{i}$ satisfy:

F.1. $\quad F_{i}$ is strictly increasing, continuous, and there is some $r_{i}>0$ such that $F_{i}(x)>x$ on $\left(0, r_{i}\right)$ and $F_{i}(x)<x$ for $x>r_{i}$.

Note that $F_{i}\left(r_{i}\right)=r_{i}$ for all $i=1, \ldots, N$. Next, assume:

F.2. $\quad r_{i} \neq r_{j}$ for $i \neq j$.

In other words, the unique positive fixed points $r_{i}$ of distinct laws of motion are all distinct. We choose the indices $i=1,2, \ldots, N$ so that

$$
r_{1}<r_{2}<\ldots<r_{N}
$$

Let $\operatorname{Prob}\left(\alpha_{n}=F_{i}\right)=p_{i}>0(i \leq i \leq N)$.

Consider the Markov process $\left\{X_{n}(x)\right\}$ with the state space $(0, \infty)$. If $y \geq r_{1}$, then $F_{i}(y) \geq$ $F_{i}\left(r_{1}\right)>r_{1}$ for $i=2, \ldots N$, and $F_{1}\left(r_{1}\right)=r_{1}$, so that $X_{n}(x) \geq r_{1}$ for all $n \geq 0$ if $x \geq r_{1}$. Similarly, if $y \leq r_{N}$, then $F_{i}(y) \leq F_{i}\left(r_{N}\right)<r_{N}$ for $i=1, \ldots, N-1$ and $F_{N}\left(r_{N}\right)=r_{N}$, so that $X_{n}(x) \leq r_{N}$ for all $n \geq 0$ if $x \leq r_{N}$. Hence, if the initial state $x$ is in $\left[r_{1}, r_{N}\right]$, then the process $\left\{X_{n}(x): n \geq 0\right\}$ remains in $\left[r_{1}, r_{N}\right]$ forever. We shall presently see that for a long run analysis we can consider $\left[r_{1}, r_{N}\right]$ as the effective state space.

We shall first indicate that on the state space $\left[r_{1}, r_{N}\right]$ the splitting condition $(H)$ is satisfied. If $x \geq r_{1}, F_{1}(x) \leq x, F_{1}^{(2)}(x) \leq F_{1}(x)$ etc. The limit of this decreasing sequence $F_{1}^{(n)}(x)$ must be a fixed point of $F_{1}$, and therefore must be $r_{1}$. Similarly, if $x \leq r_{N}$, then $F_{N}^{n}(x)$ increases to $r_{N}$. In particular,

$$
\lim _{n \rightarrow \infty} F_{1}^{(n)}\left(r_{N}\right)=r_{1}, \quad \lim _{n \rightarrow \infty} F_{N}^{(n)}\left(r_{1}\right)=r_{N}
$$

Thus, there must be a positive integer $n_{0}$ such that

$$
F_{1}^{\left(n_{0}\right)}\left(r_{N}\right)<F_{N}^{\left(n_{0}\right)}\left(r_{1}\right)
$$


This means that if $z_{0} \epsilon\left[F_{1}^{\left(n_{0}\right)}\left(r_{N}\right), F_{1}^{\left(n_{0}\right)}\left(r_{1}\right)\right]$, then

$$
\begin{aligned}
\operatorname{Prob}\left(X_{n_{0}}(x)\right. & \left.\leq z_{0} \forall x \epsilon\left[r_{1}, r_{N}\right]\right) \\
& \geq \operatorname{Pr} o b\left(\alpha_{n}=F_{1} \text { for } 1 \leq n \leq n_{0}\right)=p_{1}^{n_{0}}>0 \\
\operatorname{Prob}\left(X_{n_{0}}(x)\right. & \left.\geq z_{0} \forall x \epsilon\left[r_{1}, r_{n}\right]\right) \\
& \geq \operatorname{Pr} o b\left(\alpha_{n}=F_{N} \text { for } 1 \leq n \leq n_{0}\right)=p_{N}^{n_{0}}>0
\end{aligned}
$$

Hence, considering $\left[r_{1}, r_{N}\right]$ as the state space, and using Theorem 3.1, there is a unique invariant probability $\pi$ with the stability property holding for all initial $x \epsilon\left[r_{1}, r_{N}\right]$.

Now, define $m(x)=\min _{i=1, \ldots, N} F_{i}(x)$, and fix the initial state $x \epsilon\left(0, r_{1}\right)$.

One can verify that (i) $m$ is continuous; (ii) $m$ is strictly increasing; (iii) $m\left(r_{1}\right)=r_{1}$ and $m(x)>x$ for $x \epsilon\left(0, r_{1}\right)$, and $m(x)<x$ for $x>r_{1}$. Clearly $m^{(n)}(x)$ increases with $n$, and $m^{(n)}(x) \leq r_{1}$. The limit of the sequence $m^{(n)}(x)$ must be a fixed point, and is, therefore $r_{1}$. Since $F_{i}\left(r_{1}\right)>r_{1}$ for $i=2, \ldots, N$, there exists some $\varepsilon>0$ such that $F_{i}(y)>r_{1}(2 \leq i \leq N)$ for all $y \epsilon\left[r_{1}-\varepsilon, r_{1}\right]$. Clearly there is some $n_{\varepsilon}$ such that $m^{n_{\varepsilon}}(x) \geq r_{1}-\varepsilon$. If $\tau_{1}=\inf \left\{n \geq 1: X_{n}(x)>r_{1}\right\}$ then it follows that for all $k \geq 1$

$$
\operatorname{Prob}\left(\tau_{1}>n_{\varepsilon}+k\right) \leq p_{1}^{k}
$$

Since $p_{1}^{k}$ goes to zero as $k \rightarrow \infty$, it follows that $\tau_{1}$ is finite almost surely. Also, $X_{\tau_{1}}(x) \leq r_{N}$, since for $y \leq r_{1}$, (i) $F_{i}(y)<F_{i}\left(r_{N}\right)$ for all $\mathrm{i}$ and (ii) $F_{i}\left(r_{N}\right)<r_{N}$ for $i=1,2, \ldots, N-1$ and $F_{N}\left(r_{N}\right)=r_{N}$. (In a single period it is not possible to go from a state less than $r_{1}$ to one larger than $\left.r_{N}\right)$. By the strong Markov property, and our earlier result, $X_{\tau+m}(x)$ converges in distribution to $\pi$ as $m \rightarrow \infty$ for all $x \epsilon\left(0, r_{1}\right)$. Similarly, one can check that as $n \rightarrow \infty, X_{n}(x)$ converges in distribution to $\pi$ for all $x>r_{N}$.

Note that in growth models, the condition F.1 is often derived from appropriate "end point" or Uzawa-Inada conditions. It should perhaps be stressed that convexity assumptions have not 
appeared in the discussion of this section so far. Of course, in models of optimization, $F_{i}$ is the optimal transition of the system from one state into another, and non-convexity may lead to a failure of the splitting condition (see Majumdar, Mitra and Nyarko (1989) for details).

\subsection{One Sector Log-Cobb-Douglas Optimal Growth}

Let us recall the formulation of the one-sector growth model with a Cobb-Douglas production function $G(x)=x^{\alpha}, 0<\alpha<1$, with a representative decision maker's utility given by $u(c)=\ln c$. Following Mitra, Montrucchio and Privileggi (2004), suppose that an exogenous perturbation may reduce production by some parameter $0<k<1$ with probability $p>0$ (the same for all $t=0,1, \ldots)$. This independent and identically distributed random shock enters multiplicatively

into the production process so that output is given by $G_{r}(x)=r x^{\alpha}$ where $r \in\{k, 1\}$. The dynamic optimization problem can be explicitly written as follows:

$$
\max \mathbb{E}_{0} \sum_{t=0}^{\infty} \beta^{t} \ln c_{t}
$$

where $0<\beta<1$ is the discount factor, and the maximization is over all consumption plans $c=\left(c_{0}, c_{1}, \ldots\right)$ such that for $t=0,1,2, \ldots$

$$
c_{t}=r_{t} x_{t}^{\alpha}-x_{t+1}, \quad c_{t} \geq 0, \quad x_{t} \geq 0
$$

and $x_{0}, r_{0}$ are given.

It is well known that the optimal transition of $x_{t}$ is just described is $g(x, r)=\alpha \beta r x^{\alpha}$ i.e., the plan $x_{t}$ generated recursively by

$$
x_{t+1}=g\left(x_{t}, r_{t}\right)=\alpha \beta r_{t} x_{t}^{\alpha}
$$

is optimal.

Consider now the random dynamical system obtained by the following logarithmic transfor- 
mation of $x_{t}$ :

$$
y_{t}=-\frac{1-\alpha}{\ln k} \ln x_{t}+1+\frac{1 n \alpha+\ln \beta}{\ln k} .
$$

The new variable $y_{t}$, associated with $x_{t}$ evolves according to a linear policy, so that

$$
y_{t+1}=\alpha y_{t}+(1-\alpha)\left(1-\frac{\ln r_{t}}{\ln k}\right),
$$

which can be rewritten as

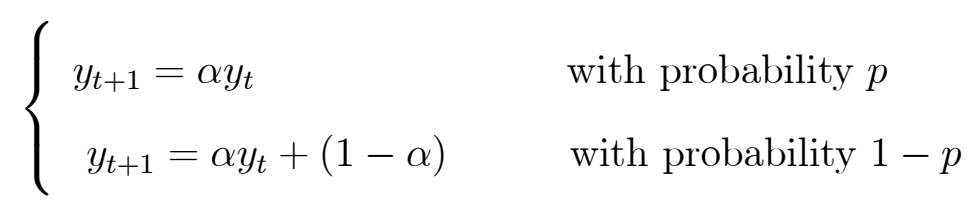

Define the maps $\gamma_{0}, \gamma_{1}$ from $[0,1]$ to $[0,1]$ by

$$
\left\{\begin{array}{c}
\gamma_{0}(y)=\alpha y \\
\gamma_{1}(y)=\alpha y+(1-\alpha)
\end{array}\right.
$$

It is useful to note here that the map $\gamma_{0}$ corresponds to the case where the shock, $r$, takes the value $k$; and the map $\gamma_{1}$ corresponds to the case where the shock, $r$, takes the value 1 . Denote $(p, 1-p)$ by $\left(p_{0}, p_{1}\right)$. Then $S=[0,1], \Gamma \equiv\left\{\gamma_{0}, \gamma_{1}\right\}$, together with $Q \equiv\left\{p_{0}, p_{1}\right\}$ is a random dynamical system. The maps $\gamma_{i}$, for $i \in\{0,1\}$, are clearly affine.

\subsubsection{The Support of the Invariant Distribution}

Let $\pi$ be the unique invariant distribution, $F_{\pi}$, its distribution function. The graphs of the functions show that for $0<\alpha<1 / 2$, the image sets of the two functions $\gamma_{0}$ and $\gamma_{1}$ are disjoint, a situation which can be described as the "non-overlapping" case. In this case, the "gap" between the two image sets (in the unit interval) will "spread" through the unit interval by successive applications of the maps (6.1). Thus, one would expect the support of the invariant distribution to be "thin" (with zero Lebesgue measure). 
On the other hand, for $1 / 2 \leq \alpha<1$, the image sets of the functions $\gamma_{0}$ and $\gamma_{1}$ have a non-empty intersection. We can refer to this as the "overlapping" case. Here, the successive iterations of the overlap can be expected to "fill up" the unit interval, so the invariant distribution should have full support.

The above heuristics are actually seen to be valid.

It is important to remark that this result does not depend on the magnitude of the discount factor $\beta$ nor on the amplitude of the shock $k$, but only on the technological parameter $\alpha$. The discount factor $\beta$ only shifts the support of the invariant distribution of the original model over the real line, while the exogenous shock $k$ affects its amplitude. The stream of research has been striving around the fundamental question on deciding for what values of $\alpha$, the invariant $F_{\pi}$ is absolutely continuous, and for what values of $\alpha, F_{\pi}$ is singular. For an exhaustive mathematical survey on the whole history of Bernoulli convolutions, see Peres, Schlag and Solomyak (1999).

It is known, in the symmetric case $p=\frac{1}{2}$, that the distribution function is "pure"; that is, it is either absolutely continuous or it is singular (Jessen and Wintner [1935]). Further, Kershner and Wintner [1935] have shown that if $0<\alpha<1 / 2$, the support of the distribution function is a Lebesgue-null Cantor set and, therefore, the distribution function is singular. For $\alpha=\frac{1}{2}$, one gets the uniform distribution, which is not singular.

For the symmetric case $p=\frac{1}{2}$, denote by $S_{\perp}$ the set of $\alpha \in(1 / 2,1)$ such that $F_{\pi}$ is singular. It was conjectured that the distribution function should be absolutely continuous with respect to Lebesgue measure when $1 / 2<\alpha<1$. Wintner [1935] showed that if $\alpha$ is of the form $(1 / 2)^{1 / k}$ where $k \in\{1,2,3, \ldots\}$, then the distribution function is absolutely continuous. However, in the other direction, Erdös [1939] showed that when $\alpha$ is the positive solution of the Equation $\alpha^{2}+\alpha-1=0$, so that $\alpha=(\sqrt{5}-1) / 2$, then $\alpha \in S_{\perp}$.

Erdös also showed that $S_{\perp} \cap(\xi, 1)$ has zero Lebesgue measure for some $\xi<1$, so that absolute continuity of the invariant distribution obtains for (almost every) $\alpha$ sufficiently close to 1. A conjecture that emerged from these findings is that the set $S_{\perp}$ itself should have Lebesgue measure zero. In their brief discussion of this problem, Dubins and Freedman [1966] state that 
deciding whether the invariant distribution is singular or absolutely continuous for $\alpha>1 / 2$ is a "famous open question".

Solomyak [1995] made a real breakthrough when he showed that $S_{\perp}$ has zero Lebesgue measure. More precisely, he established that for almost every $\alpha \in(1 / 2,1)$, the distribution has density in $L^{2}(R)$ and for almost every $\alpha \in\left(2^{-1 / 2}, 1\right)$ the density is bounded and continuous. A simpler proof of the same result was subsequently presented by Peres and Solomyak [1996].

More recent contributions to this literature deal with the asymmetric case $p \neq 1 / 2$. (see, for example, Peres and Schlag (2000)). For example, $F_{\pi}$ is singular for values of parameters $(\alpha, p)$ such that $0<\alpha<p^{p}(1-p)^{(1-p)}$, while $F_{\pi}$ is absolutely continuous for almost every $p^{p}(1-p)^{(1-p)}<\alpha<1$ whenever $1 / 3 \leq p \leq 2 / 3$. For more details see Mitra, Montrucchio and Privileggi (2004). 


\section{References}

Athreya, K.B. and Bhattacharya, R.N. (2000): "Random Iteration of I.I.D. Quadratic Maps" in Stochastics in Finite and Infinite Dimensions: in Honor of Gopinath Kallianpur (eds. T. Hida et. al.), Birkhauser, Basel, pp. 49-58.

Athreya, K. and M. Majumdar (2002): "Estimating the Stationary Distribution of a Markov Chain", Economic Theory, 27, pp. 729-742.

Athreya, K.B. (2004): "Stationary Measures for Some Markov Chain Models in Ecology and Economics", Economic Theory, vol. 23, pp. 107-122.

Bhattacharya, R.N. and O. Lee (1988): "Asymptotics of a Class of Markov Processes, That Are Not in General Reducible", Annals of Probability, 16, pp. 1333-47.

Bhattacharya, R.N. and B.V. Rao (1993): "Random Iterations of Two Quadratic Maps" in Stochastic Processes (eds. S. Cambanis, J.K. Ghosh, R.L. Karandikar and P.K. Sen), Springer Verlag, New York, pp. 13-21.

Bhattacharya, R.N. and M. Majumdar (1999): "On a Theorem of Dubins and Freedman", Journal of Theoretical Probability, 12, pp. 1067-1087.

Bhattacharya, R.N. and M. Majumdar (2001): "On a Class of Stable Random Dynamical Systems: Theory and Applications", Journal of Economic Theory, 96, pp. 208-229.

Bhattacharya, R.N. and Majumdar, M. (2007): "Random Iterates of Monotone Maps", Submitted to Review of Economic Design.

Bhattacharya, R. and Majumdar, M. (2007) Random Dynamical Systems: Theory and Applications, Cambridge University Press, Cambridge.

Chakraborty, S. and Rao, B.V. (1998): "Completeness of Bhattacharya Metric on the Space of Probabilities", Statistics and Probability Letters, 36, pp. 321-326.

Diaconis, P. and D. Freedman (1999): "Iterated Random Functions", SIAM Review, 41, 45-79.

Dubins, L.E. and D. Freedman (1966): "Invariant Probabilities for Certain Markov Processes", Annals of Mathematical Statistics, 37, pp. 837-858. 
Ellner, S. (1984): "Asymptotic Behavior of Some Stochastic Difference Equation Population Models", Journal of Mathematical Biology, 19, pp. 169-200.

Erdös, P. (1939): "On a Family of Symmetric Bernoulli Convolutions", American Journal of Mathematics, 61, pp. 944-975.

Goswami, A. (2004): "Random Continued Fractions: A Markov Chain Approach", Economic Theory, 23, pp. 85-106.

Jessen, B. and Wintner, A. (1935): Distribution Function and the Rieman Zeta Function, Transactions of the American Mathematical Society, 38, pp. 48-58.

Kershner, R. and Wintner, A. (1935): "On Symmetric Bernoulli Convolutions", American Journal of Mathematics, 57, pp. 541-548.

Loeve, M. (1963): Probability Theory, Van Nostrand, Princeton.

Majumdar, M., Mitra, T., and Nyarko, Y. (1989): "Dynamic Optimization under Uncertainty: Non-convex Feasible Set" in Joan Robinson and Modern Economic Theory (ed. G.R. Feiwel), MacMillan, London, pp. 545-590.

Mitra, K. (1998): "On Capital Accumulation Paths in Neoclassical Models in Stochastic Growth Models", Economic Theory, 11, pp. 457-464.

Mitra, T., Montrucchio, L. and Privileggi, F. (2004): "The Nature of the Steady State in Models of Optimal Growth under Uncertainty", Economic Theory, 23, pp. 39-71.

Montrucchio, L. and Privileggi, F. (1999): "Fractal Steady States in Stochastic Optimal Control Models", Annals of Operations Research, 88, pp. 183-197.

Orey, S. (1971): Limit Theorems for Markov Chain Probabilities, Van Nostrand, New York.

Peres, Y., Shlag, W. and Solomyak, B. (1999): "Sixty Years of Bernoulli Convolutions", in Fractal Geometry and Stochastics, 2, (C. Bandt, S. Graf, M. Zahle, eds.), Birkhauser, Basel, pp. 39-65.

Peres, Y. and Solomyak, B. (1996). "Absolute Continuity of Benoulli Convolutions, A Simple Proof", Mathematical Research Letters, 3, pp. 231-239.

Peres, Y. and Schlag, W. (2000): "Smoothness of Projections. Bernoulli Convolutions and 
the Dimension of Exception", Duke Mathematical Journal, 102, pp. 193-251.

Solomyak, B. (1995): "On the Random Series $\sum \pm \lambda^{n}$ : an Erdös Problem", Annals of Mathematics, 142, pp. 611-625.

Solow, R.M. (1956): "A Contribution of the Theory of Economic Growth", Quarterly Journal of Economics, 70, pp. 65-94.

Wintner (1935): "On Convergent Poisson Convolutions", American Journal of Mathematics, 57, pp. $827-838$.

Yahav, J.A. (1975): "On a Fixed Point Theorem and Its Stochastic Equivalent", Journal of Applied Probability, vol. 12, pp. 605-611. 